\title{
Directrices en la decisión del manejo quirúrgico en la cirugía de columna vertebral
}

\author{
Guidelines in the decision of surgical management in spine surgery
}

José M. ${ }^{a}$ Jiménez-Ávila1*, Omar Sánchez-García y Arelhi C. González-Cisneros ${ }^{3}$

${ }^{1}$ Clínica de Columna, Centro Médico Nacional de Occidente, Instituto Mexicano del Seguro Social; ${ }^{2}$ Hospital Militar Regional de Especialidades;

${ }^{3}$ Escuela de Medicina del Instituto Tecnológico y de Estudios Superiores de Monterrey, Campus Guadalajara. Guadalajara, Jalisco, México

\section{Resumen}

Introducción: El cirujano de columna cuenta con conocimientos y habilidades que le permiten tomar decisiones al momento de realizar un procedimiento quirúrgico, basado en la evidencia, en valores éticos y en las expectativas del paciente. Cualquier procedimiento quirúrgico será precedido por el diagnóstico basado en cuatro pilares fundamentales: traumático, degenerativo, síndrome de destrucción vertebral y deformidades. Una vez categorizada la lesión, el cirujano utilizará sus conocimientos para identificar la inestabilidad o el compromiso neurológico. Al realizar un procedimiento quirúrgico, en el transoperatorio deberán tenerse en mente los cuatro objetivos fundamentales con los que contará para lograr un manejo satisfactorio: descomprimir, instrumentar, fusionar y corregir el balance sagital. Objetivo: Analizar los criterios fundamentales en la toma de decisión para el manejo conservador o quirúrgico en la columna vertebral. Resultados: Se analizaron las escalas de medición y de evaluación más frecuentes y mayormente efectivas en relación a la cirugía de columna, mismos que obligadamente tienen que ser aplicadas y evaluadas en cada caso particular, lo cual permite fortalecer la impresión diagnóstica y el valor pronóstico. Conclusión: Implementar la cultura del uso de mediciones de resultados de pacientes como instrumento de evaluación ayuda al cirujano de columna a decidir un plan de tratamiento que pueda adecuarse a las preferencias y las necesidades del paciente. Debemos fundamentar nuestro objetivo clínico en la estabilidad y el compromiso neurológico del paciente, existiendo cuatro posibilidades diagnósticas, que se corregirán para cuatro objetivos fundamentales.

PALABRAS CLAVE: Cirugía. Columna. Guías de práctica clínica. Indicaciones. Vertebral.

\section{Abstract}

Introduction: The spine surgeon has knowledge and skills that allow him to make decisions when performing a surgical procedure, based on the evidence and ethical values and expectations of the patient. Any surgical procedure will be preceded by a diagnosis based on four fundamental pillars: traumatic, degenerative, vertebral destruction syndrome and deformities. Once the lesion is categorized, the surgeon will use his knowledge to identify instability or neurological compromise. When performing a surgical procedure, it should be kept in mind in the transoperative the four key objectives that will count, to achieve a satisfactory management: decompress, implement, merge and correct the sagittal balance. Objective: To analyze the fundamental criteria in decision making, for conservative or surgical management in the spine. Results: The assessment scales and the most frequent and best practices in relation to spine surgery will be analyzed, as well as the applications that will be applied and evaluated in each particular case, which will strengthen the diagnostic impression and the value forecast.

\author{
Correspondencia: \\ *José María Jiménez Ávila \\ Belisario Domínguez, 1000 \\ Col. Independencia \\ C.P. 44340, Guadalajara, Jal., México \\ E-mail: josemajimeneza@ hotmail.com
}

Fecha de recepción: 06-07-2018

Fecha de aceptación: 01-02-2019

DOI: 10.24875/CIRU.19000583
Cir Cir. 2019;87:299-307

Contents available at PubMed www.cirugiaycirujanos.com 
Conclusion: Implementing the culture of using patient outcome measurements as an assessment tool helps the spine surgeon to decide on a treatment plan that can be adapted to the patient's preferences and needs. We must base our clinical objective on the stability and the neurological commitment of the patient, there being four diagnostic possibilities; which will be corrected for four fundamental objectives.

KEY WORDS: Clinical practice guidelines. Indications. Spine. Surgery. Vertebral.

\section{Introducción}

El cirujano de columna cuenta con una serie de conocimientos y habilidades que le permiten tomar decisiones al momento de realizar un procedimiento quirúrgico, basado en la evidencia, haciendo uso consciente explícito y juicioso de la mejor evidencia científica disponible, para tomar decisiones sobre sus pacientes, que se sustentarán en su experiencia clínica individual, en los valores éticos y en las expectativas del paciente.

En su arsenal científico gira una gran cantidad de información, la cual tiene que seleccionar para tomar la mejor decisión, pero siempre debe tener en mente algunos principios fundamentales que nunca debe olvidar cuando decida realizar una cirugía'. Lo primero que debe determinar antes de pensar en cualquier procedimiento quirúrgico es el diagnóstico, que estará basado en cuatro pilares: traumático, degenerativo, síndrome de destrucción vertebral y deformidades (Fig. 1). Una vez categorizada la lesión, el cirujano debe utilizar sus conocimientos para identificar datos de inestabilidad o compromiso neurológico. Para ello se auxiliará de estudios de gabinete, como la radiografía simple, la cual, mediante mediciones específicas, indicará si la columna mantiene un equilibrio o bien está compensada; posteriormente se complementará con estudios de tomografía computarizada y resonancia magnética, que confirmarán o descartarán datos compresivos, de acuerdo con un nivel específico, lo que le permitirá identificar el lugar de la posible corrección, teniendo en mente el principio y la técnica que utilizará.

El método de referencia para el tratamiento de las lesiones es mantener la estabilidad, la descompresión de las estructuras neurales, la restauración de la capacidad de carga y la restitución de las curvas fisiológicas de la columna vertebral (equilibrio), lo que permite una rehabilitación más rápida y evita el desarrollo de deformidades o lesiones subsecuentes² (Fig. 2).

Para realizar el procedimiento quirúrgico deberá tener en mente en el transoperatorio los cuatro «principios quirúrgicos» con los que contará para lograr un

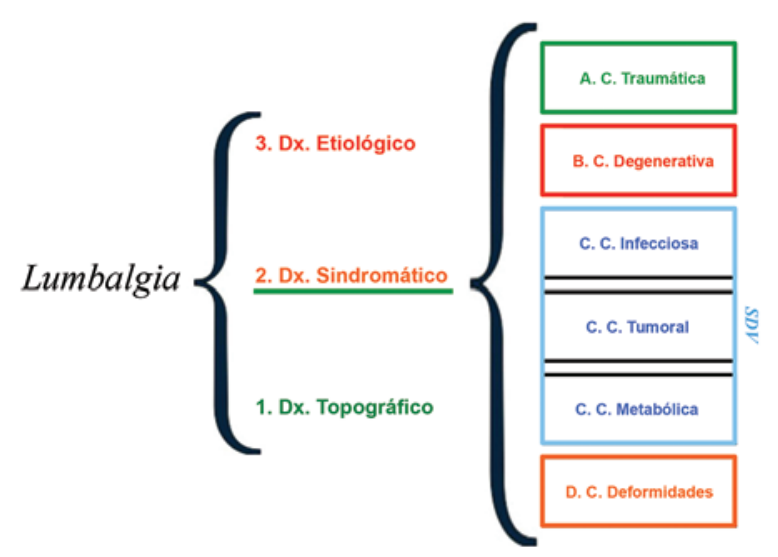

Figura 1. Diagnóstico basado en el algoritmo de referencia para lumbalgia.

manejo satisfactorio: descomprimir, instrumentar, fusionar y corregir el balance sagital (Fig. 3).

\section{Objetivo}

Analizar los criterios fundamentales en la toma de decisión para la realización de una cirugía en la columna vertebral.

\section{Método}

El cirujano de columna debe conocer las siguientes herramientas para el buen diagnóstico y seguimiento de los pacientes con patología de columna.

Es importante que el cirujano de columna evidencie sus resultados, tanto antes como después de la cirugía, utilizando la evidencia radiográfica, mediante el uso de mediciones espinopélvicas y de alineación sagital $^{3}$ (Fig. 4).

\section{Usuarios}

Cirujanos de columna (ortopedistas o neurocirujanos) involucrados en la atención de pacientes con patología de columna vertebral, asignados a tercer nivel de atención. 


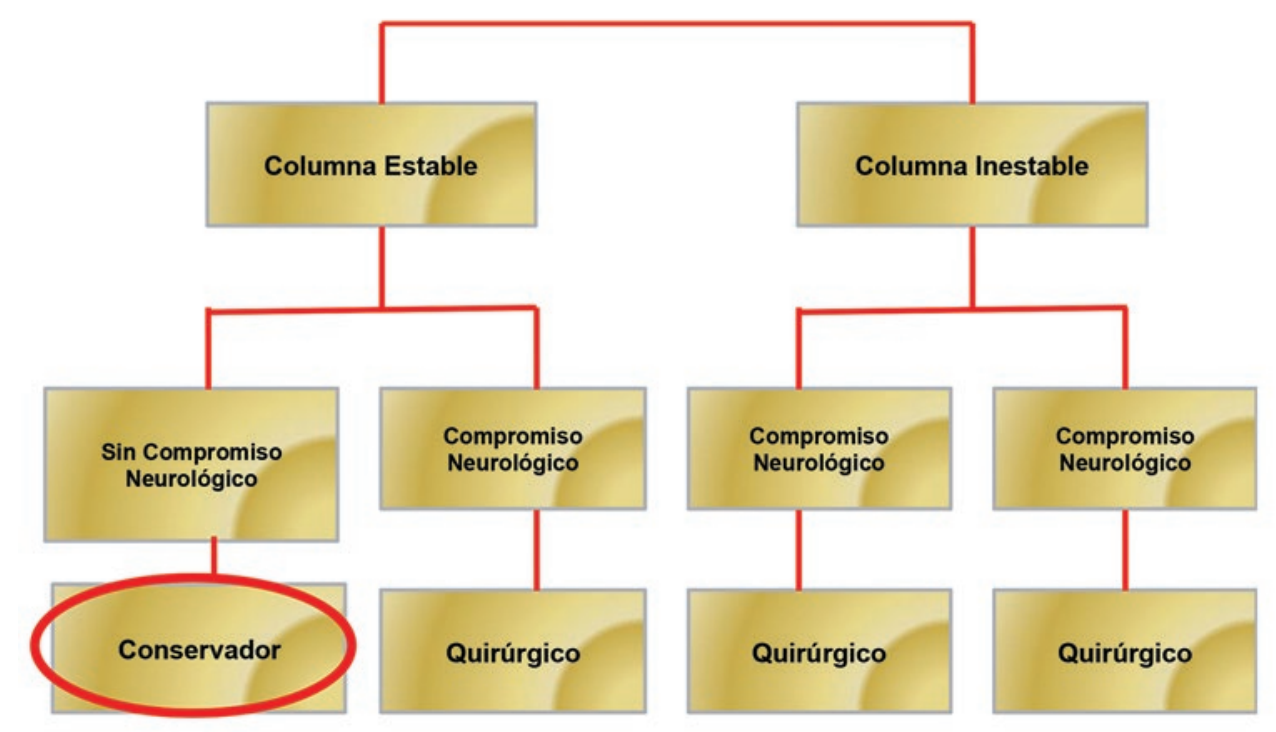

Figura 2. Criterios de estabilidad y compresión para decisión quirúrgica.

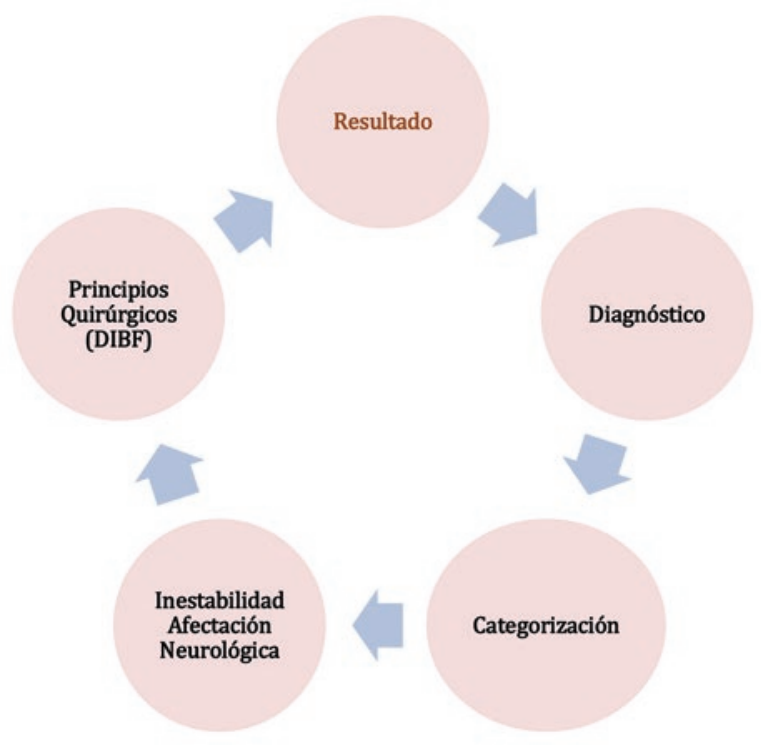

Figura 3. Diagnóstico, Categorización, Inestabilidad, Afectación Neurológica, Principios quirúrgicos y Resultado.

\section{Población diana}

Hombres y mujeres con un diagnóstico asociado a patologías de la columna vertebral.

\section{Selección de la evidencia}

Se hizo una búsqueda dividiendo las enfermedades de la columna en degenerativas, traumáticas, síndrome de destrucción vertebral y deformidades. Las palabras clave utilizadas y traducidas del español al
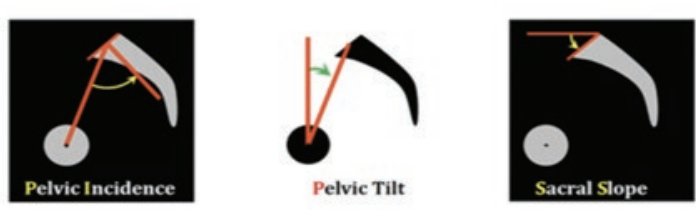

$$
\mathrm{PI}=\mathbf{P T}+\mathbf{S S}
$$
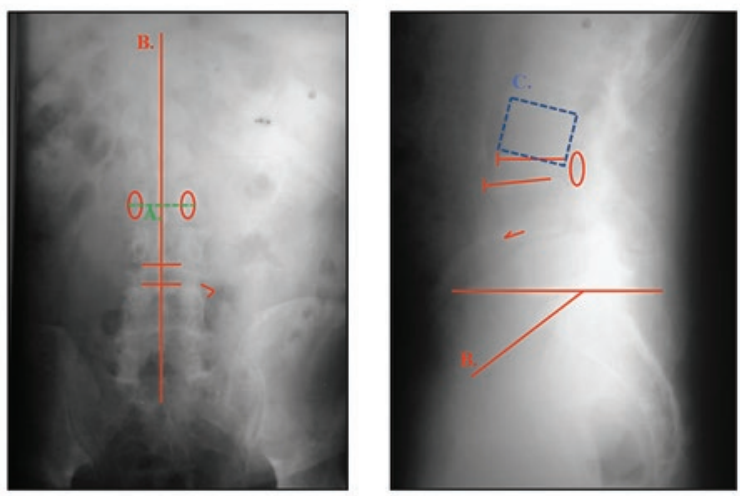

Figura 4. Criterios radiográficos (mediciones PI, PT, SS, lordosis, listesis, etc.)

inglés por el diccionario de vocabulario controlado utilizado para indexar artículos en PubMed, MeSH, fueron: fracture; stability; spinopelvic; clinical practice guidelines; cervical, thoracic and lumbar low back pain; neoplasms; infections; discitis; osteoporosis; stenosis; hernia y listhesis. Las bases de datos consultadas fueron PubMed y Cochrane principalmente, con el apoyo de otras como Scopus y Lilacs. Se encontraron 16 artículos, de los cuales se tomaron siete. 
Tabla 1. Evidencia de estudios de gabinete en lesiones traumáticas

\begin{tabular}{|c|c|c|c|}
\hline \multicolumn{4}{|l|}{ A. Fracturas } \\
\hline $\begin{array}{l}\text { American Spinal Injury } \\
\text { Association (ASIA) }\end{array}$ & A & C & $\mathrm{E}$ \\
\hline $\begin{array}{l}\text { Magerl Asociación de } \\
\text { Osteosíntesis } \\
\text { (Magerl AO) }\end{array}$ & A & B & $\mathrm{C}$ \\
\hline $\begin{array}{l}\text { Thoracolumbar injury } \\
\text { classification and } \\
\text { severity score (TLICS) }\end{array}$ & $0-3$ & 4 & $>5$ \\
\hline \multicolumn{4}{|c|}{ Load-Sharing (McCormack) } \\
\hline Daño cuerpo & $1=<30 \%$ & $2=30-60 \%$ & $3=>60 \%$ \\
\hline Fragmento en canal & $1=0-1 \mathrm{~mm}$ & $2=2 \mathrm{~mm}$ & $3=>2 \mathrm{~mm}$ \\
\hline Corrección de cifosis & $1=3^{\circ}$ & $2=4-9^{\circ}$ & $3=>10^{\circ}$ \\
\hline Resonancia magnética & $0=\sin$ daño & \multicolumn{2}{|c|}{$1=$ con daño } \\
\hline
\end{tabular}

\begin{tabular}{lc} 
A. Lesión subaxial (SLIC) & \\
\hline Morfofisiología & Puntos \\
\hline Sin anormalidades & 0 \\
Compresión & 1 \\
Estallido & 3 \\
Distracción & 4 \\
Rotación/traslación & Puntos \\
\hline Complejo discoligamentario & 0 \\
\hline Intacto & 1 \\
Indeterminado & 2 \\
Roto & Puntos \\
\hline Estatus neurológico & 0 \\
\hline Intacto & 1 \\
Lesión radicular & 2 \\
Lesión medular completa & 3 \\
Lesión medular incompleta & 1 \\
Neuromodificador & Total \\
\hline
\end{tabular}

Las fuentes de información databan entre los años 2008 y 2018.

Los integrantes del grupo constructor de esta guía localizaron y analizaron el material, cuya inclusión final se realizó por medio de una discusión entre los mismos participantes utilizando los criterios de las guías para los usuarios de la literatura médica publicados en PubMed entre los años 2010 y 2018.

\section{Criterios de evidencia}

\section{A. Columina traumática (verde)}

La evidencia indica que el tratamiento de las fracturas vertebrales se basa en las opciones de ser un
Tabla 2. Evidencia de estudios de gabinete en lesiones degenerativas cervicales

\begin{tabular}{lc}
\hline A. Lesión subaxial (SLIC) & Puntos \\
\hline Morfofisiología & 0 \\
\hline Sin anormalidades & 1 \\
Compresión & 1 \\
Estallido & 3 \\
Distracción & 4 \\
Rotación/traslación & Puntos \\
\hline Complejo discoligamentario & 0 \\
\hline Intacto & 1 \\
Indeterminado & 2 \\
Roto & Puntos \\
\hline Estatus neurológico & 0 \\
\hline Intacto & 1 \\
Lesión radicular & 2 \\
Lesión medular completa & 3 \\
Lesión medular incompleta & 1 \\
Neuromodificador & Total \\
\hline
\end{tabular}

\section{B. Degenerativo cervical}

\begin{tabular}{|c|c|c|c|}
\hline Radiografía AP & Sí No & Radiografía lateral & Sí No \\
\hline Estrechez del espacio discal & & Inestabilidad & \\
\hline Presencia de osteofitos & & Presencia de osteofitos & \\
\hline Deformidad espinal & & Pérdida de la lordosis & \\
\hline
\end{tabular}

\begin{tabular}{|c|c|c|c|}
\hline RM sagital & Sí No & RM axial & Sí No \\
\hline Cambios degenerativos & & $\begin{array}{l}\text { Cambios } \\
\text { degenerativos }\end{array}$ & \\
\hline $\begin{array}{l}\text { Compresión de raíces } \\
\text { nerviosas }\end{array}$ & & $\begin{array}{l}\text { Compresión de raíces } \\
\text { nerviosas }\end{array}$ & \\
\hline $\begin{array}{l}\text { Disminución del espacio } \\
\text { intervertebral }\end{array}$ & & $\begin{array}{l}\text { Engrosamiento de las } \\
\text { láminas }\end{array}$ & \\
\hline Listesis & & $\begin{array}{l}\text { Estenosis en recesos y } \\
\text { forámenes }\end{array}$ & \\
\hline Protrusiones discales & & $\begin{array}{l}\text { Hipertrofia de } \\
\text { ligamento amarillo }\end{array}$ & \\
\hline Formación de osteofitos & & Hipertrofia facetaria & \\
\hline Desmielinización & & Líquido interarticular & \\
\hline \multicolumn{4}{|l|}{ Edema } \\
\hline \multicolumn{4}{|l|}{ Gliosis } \\
\hline Isquemia & & & \\
\hline Mielomalacia & & & \\
\hline
\end{tabular}


Tabla 3. Evidencia de estudios de gabinete en lesiones degenerativas lumbares

\begin{tabular}{|c|c|c|c|c|c|}
\hline \multicolumn{6}{|l|}{ B. Degenerativo lumbar } \\
\hline Radiografía AP & Sí & No & Radiografía lateral & Sí & No \\
\hline Asimetría pedicular & & & Ausencia de foramen & & \\
\hline Megapofisis de L5 & & & Deterioro facetario & & \\
\hline Osteofitos laterales & & & Disminución del espacio intervertebral & & \\
\hline Pinzamiento asimétrico & & & Listesis & & \\
\hline Pérdida del eje & & & Osteofitos anteriores & & \\
\hline Rotoescoliosis degenerativa & & & Pérdida de la lordosis & & \\
\hline Sacralización L5 & & & Pinzamiento discal & & \\
\hline TC axial & Sí & No & TC tejidos blandos & Sí & No \\
\hline Engrosamiento laminar & & & Engrosamiento ligamento amarillo & & \\
\hline Hipertrofia facetaria & & & Sinovitis articular & & \\
\hline Estenosis en recesos y foramen & & & Protrusión discal & & \\
\hline \multicolumn{6}{|l|}{ Osteofitos posteriores } \\
\hline \multicolumn{6}{|l|}{$<11 \mathrm{~mm}$ conducto medular } \\
\hline \multicolumn{6}{|l|}{ Orientación interlínea articular } \\
\hline RM sagital & Sí & No & RM axial & Sí & No \\
\hline Cambios degenerativos & & & Compresión de raíces nerviosas & & \\
\hline Compresión de raíces nerviosas & & & Cambios degenerativos & & \\
\hline Disminución del espacio intervertebral & & & Engrosamiento de las láminas & & \\
\hline Listesis & & & Estenosis en recesos y forámenes & & \\
\hline \multirow[t]{3}{*}{ Protrusiones discales } & & & Hipertrofia de ligamento amarillo & & \\
\hline & & & Hipertrofia facetaria & & \\
\hline & & & Líquido interarticular & & \\
\hline
\end{tabular}

AP: anteroposterior; RM: resonancia magnética; TC: tomografía computarizada.

tratamiento conservador o quirúrgico, apoyándose este último en determinantes tanto clínicos como radiológicos ${ }^{4}$ (Tabla 1).

\section{B. Columina degenerativa (rojo)}

El proceso degenerativo debe ser evidenciado de manera específica, ya que cada dato puede ser un determinante para la decisión de una posible cirugía, por lo que debe identificarse cada una de las lesiones, tanto radiográficas como de resonancia magnética (Modic y Pfirrmann) ${ }^{5-7}$ (Fig. 5).

\section{Cervical (Tabla 2) Lumbar (Tabla 3)}

\section{SíndRome de destrucción vertebral (azUL)}

Este es un síndrome de difícil estudio, lo que genera la necesidad de determinar patrones patológicos y sus características propias de los segmentos afectados, por lo que al decidir deberá evidenciar si existe una alteración en la forma de la vértebra provocada por una destrucción, ya que es importante determinar su etiología, estabilidad y posible compresión neurológica, y para tal efecto puede utilizarse la clasificación SINS (Spinal Instability Neoplastic Score), que considera lesión estable (0-6 puntos), lesión potencialmente inestable (7-12 puntos) y lesión definitivamente inestable (13-18 puntos) $)^{8,9}$.

En caso de ser una lesión tumoral, la clasificación WBB (Weinstein, Boriani, Biagini) será de utilidad para estadificar y establecer un posible tratamiento ${ }^{10}$.

Es obligatorio estudiar a los pacientes bajo tres perspectivas (Tabla 4):

C.1. Columna infecciosa.

C.2. Columna tumoral.

C.3. Columna metabólica.

\section{Columna deformidades (naranja)}

Las deformidades son una alteración idiopática 0 adquirida, y lo que determina su manejo debe estar 
Tabla 4. Evidencia de estudios de gabinete en lesiones de destrucción vertebral

\begin{tabular}{|c|c|c|}
\hline \multicolumn{3}{|l|}{ C. Destrucción vertebral } \\
\hline Localización & Puntos & \\
\hline En la unión (occipital-C2, C7-T2, T11-L1, L5-S1) & 3 & \\
\hline Columna móvil (C3-C6, L2-L4) & 2 & \\
\hline Semirrígida (T3-T10) & 1 & \\
\hline Rígida (S2-S5) & 0 & \\
\hline Alivio del dolor en decúbito o reposo & Puntos & \\
\hline Sí & 3 & \\
\hline No (dolor ocasional, pero no mecánico) & 1 & \\
\hline Lesión libre de dolor & 0 & \\
\hline Lesión ósea & Puntos & \\
\hline Lítica & 2 & \\
\hline Mixta (lítica/blástica) & 1 & \\
\hline Blástica & 0 & \\
\hline Alineación radiográfica de la columna & Puntos & \\
\hline Subluxación, traslación presente & 4 & \\
\hline Deformidad de novo (cifosis, escoliosis) & 2 & \\
\hline Alineación anormal & 0 & \\
\hline Colapso del cuerpo vertebral & Puntos & \\
\hline$>50 \%$ & 3 & \\
\hline$<50 \%$ & 2 & \\
\hline No hay colapso, con > 50\% del cuerpo involucrado & 1 & \\
\hline Ninguna de las anteriores & 0 & \\
\hline Compromiso de los elementos posterolaterales & Puntos & \\
\hline Bilateral & 3 & \\
\hline Unilateral & 1 & Total SINS \\
\hline Ninguno de los anteriores & 0 & \\
\hline Clasificación WBB & A B CDE & \\
\hline Extensión & $1-12$ & \\
\hline
\end{tabular}

SINS: Spinal Instability Neoplastic Score; WBB: Weinstein, Boriani, Biagini.

Tabla 5. Evidencia de estudios de gabinete en deformidades

D. Deformidades

\begin{tabular}{|c|c|c|c|c|}
\hline Edad & \multicolumn{3}{|c|}{$1=<20$} & $2=>21$ \\
\hline Ángulo de Cobb & \multicolumn{3}{|l|}{$1=<40^{\circ}$} & $2=>41^{\circ}$ \\
\hline Risser & 1 & 3 & 4 & 6 \\
\hline Clasificación de Lenke & 1 & 3 & 4 & 6 \\
\hline Modificador lumbar Lenke & \multicolumn{2}{|l|}{ A } & B & C \\
\hline Modificador sagital Lenke & Positivo (+) & & N & Negativo (-) \\
\hline
\end{tabular}

regido por la angulación de la deformidad y secundariamente por la situación clínica"11 (Tabla 5).

\section{Índice de discapacidad}

El índice de discapacidad de Oswestry se ha convertido en una de las medidas específicas de la evolución de los pacientes con patología espinal. Esta evaluación muestra datos que permiten medir y validar la percepción de la enfermedad por parte del 

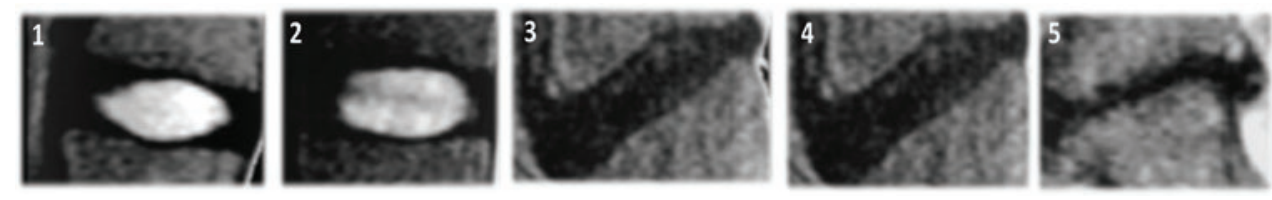

Figura 5. Clasificación de la enfermedad degenerativa del disco lumbar (Pfirmann).

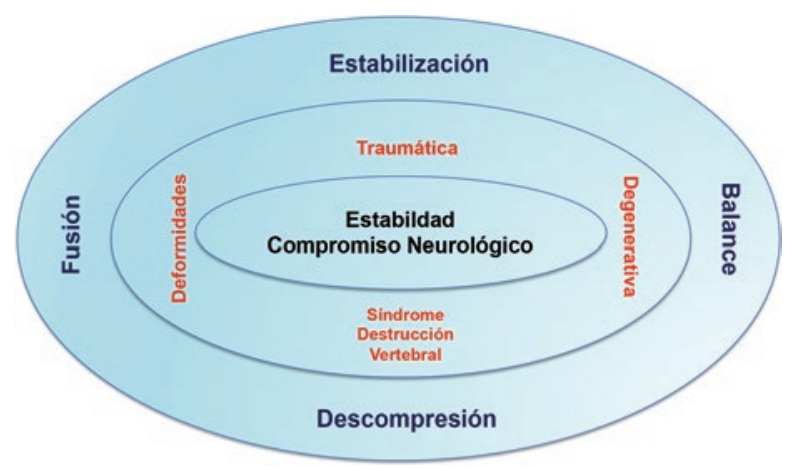

Figura 6. Directrices para la decisión de un procedimiento quirúrgico en columna.

paciente, así como identificar sus expectativas para continuar con el tratamiento ${ }^{12-15}$ (Tabla 6).

\section{Discusión}

La cirugía de columna, en la actualidad altamente especializada, se considera un procedimiento que requiere ser evaluado tanto antes como después de la intervención.

Evaluar de manera sistematizada los resultados radiológicos de los procedimientos quirúrgicos relacionados con la columna vertebral favorece el seguimiento y la posible detección temprana de complicaciones 0 un buen seguimiento satisfactorio de la cirugía.

En un estudio realizado por Falavigna, et al..$^{12}$, en el que se hizo una entrevista electrónica a 1635 cirujanos de columna de todo el mundo, el $31.9 \%$ respondieron que no utilizaban una evaluación de manera sistemática, siendo la principal barrera la sistematización de los datos. La evaluación suele ser más frecuente en los EE.UU. y en Europa, y es menos utilizada en Asia-Pacífico y Latinoamérica, lo cual puede deberse a situaciones de índole cultural.

La mayoría de los cirujanos de columna desconocen las medidas de resultados radiográficos en el cuidado de la columna vertebral. Se sabe que uno de cada tres cirujanos de columna no utilizan de manera sistemática las mediciones de resultados de los pacientes, y que uno de cada cuatro no están familiarizados con algún tipo de clasificación ${ }^{12}$. Las causas probables son la falta de tiempo, la falta de colaboración por parte del personal en la recolección de los datos y la falta de implementación en la formación de los nuevos cirujanos. Por otro lado, una de las barreras al utilizar cualquier tipo de evaluación es que se utilizan distintos registros o evaluaciones en las diferentes partes del mundo, siendo la tendencia actual la sistematización del seguimiento. Para lograr su utilización de manera generalizada es importante establecer estrategias para fortalecer la educación y poder conducir al incremento en la utilización de estas mediciones (Tabla 7). A su vez, es crucial contar con una base de datos sistematizada y codificada para poder construir los registros, y tener un sistema de evaluación obligado para aplicarlo en cualquier momento de la evaluación del paciente.

\section{Conclusión}

Implementar la cultura del uso de mediciones de resultados en pacientes como instrumento de evaluación ayuda al cirujano de columna a decidir un plan de tratamiento que pueda adecuarse a las preferencias y las necesidades del paciente, mejorar el manejo y monitorear el progreso de su tratamiento.

Los beneficios van desde evaluar cuantitativamente las características de la enfermedad, monitorizar y comparar resultados de tratamiento entre diferentes centros, con el objeto de optimizar los recursos, hasta generar un análisis económico de los cuidados de las patologías de columna. Además, conocer estos resultados bajo una escala numérica facilita que se detecten los cambios entre los periodos preoperatorio y posoperatorio.

En resumen, al enfrentarnos a una patología de columna debemos fundamentar nuestro objetivo clínico en conceptos como la estabilidad y el compromiso neurológico del paciente. Para ello existirán cuatro posibilidades diagnósticas, mismas que se corregirán por cuatro objetivos fundamentales, y las decisiones quirúrgicas deben basarse siempre en el resultado de la evidencia (Fig. 6). 
Tabla 6. Test de evaluación funcional de Oswestry

\begin{tabular}{|c|c|c|c|}
\hline 1 & Intensidad del dolor & 2 & Actividades de la vida cotidiana (lavarse, vestirse) \\
\hline & Actualmente no tengo dolor de columna ni de pierna & & Las realizo sin ningún dolor \\
\hline & Mi dolor de columna o pierna es muy leve en este momento & & Puedo hacer de todo en forma normal, pero con dolor \\
\hline & Mi dolor de columna o pierna es moderado en este momento & & Las realizo en forma más lenta y cuidadosa por el dolor \\
\hline & Mi dolor de columna o pierna es intenso en este momento & & Ocasionalmente requiero ayuda \\
\hline & Mi dolor de columna o pierna es muy intenso en este momento & & Requiero ayuda a diario \\
\hline & Mi dolor es el peor imaginable en este momento & & Necesito ayuda para todo, estoy postrado en cama \\
\hline \multirow[t]{7}{*}{3} & Levantar objetos & 4 & Caminar \\
\hline & Puedo levantar objetos pesados desde el suelo sin dolor & & Camino todo lo que quiero sin dolor \\
\hline & Puedo levantar objetos pesados desde el suelo, pero con dolor & & No puedo caminar más de 1-2 km debido al dolor \\
\hline & $\begin{array}{l}\text { No puedo levantar objetos pesados del suelo debido al dolor, pero } \\
\text { sí cargar un objeto pesado desde una mayor altura, p. ej. desde } \\
\text { una mesa }\end{array}$ & & No puedo caminar más de 500-1000 m debido al dolor \\
\hline & Solo puedo levantar desde el suelo objetos de peso mediano & & No puedo caminar más de 500 m debido al dolor \\
\hline & Solo puedo levantar desde el suelo cosas muy livianas & & Solo puedo caminar ayudado por uno o dos bastones \\
\hline & No puedo levantar ni cargar nada & & Estoy prácticamente en cama, me cuesta mucho ir al baño \\
\hline \multirow[t]{7}{*}{5} & Sentarse & 6 & Pararse \\
\hline & $\begin{array}{l}\text { Me puedo sentar en cualquier silla todo el rato que quiera sin sentir } \\
\text { dolor }\end{array}$ & & Puedo hacer de todo solo y en forma normal, pero con dolor \\
\hline & Solo en un asiento especial puedo sentarme sin dolor & & Puedo permanecer de pie lo que quiero, aunque con dolor \\
\hline & No puedo estar sentado más de 1 hora sin dolor & & No puedo estar más de 1 hora parado libre de dolor \\
\hline & No puedo estar sentado más de 30 minutos sin dolor & & No puedo estar parado más de 30 minutos libre de dolor \\
\hline & No puedo permanecer sentado más de 10 minutos sin dolor & & No puedo estar parado más de 10 minutos sin dolor \\
\hline & No puedo permanecer ningún instante sentado sin que sienta dolor & & No puedo permanecer ningún instante de pie sin dolor \\
\hline \multirow[t]{7}{*}{7} & Dormir & 8 & Actividad sexual \\
\hline & Puedo dormir bien, libre de dolor & & Normal sin dolor de columna \\
\hline & Ocasionalmente el dolor me altera el sueño & & Normal, aunque con dolor ocasional de columna \\
\hline & Por el dolor no logro dormir más de 6 h seguidas & & O así normal, pero con importante dolor de columna \\
\hline & Por el dolor no logro dormir más de $4 \mathrm{~h}$ seguidas & & Seriamente limitada por el dolor de la columna \\
\hline & Por el dolor no logro dormir más de $2 \mathrm{~h}$ seguidas & & O así sin actividad por el dolor de columna \\
\hline & No logro dormir nada sin dolor & & Sin actividad debido a los dolores de columna \\
\hline \multirow[t]{12}{*}{9} & Actividades sociales (fiestas, deportes) & 10 & Viajar \\
\hline & Sin restricciones, libres de dolor & & Sin problema, libre de dolor \\
\hline & Mi actividad es normal, pero aumenta el dolor & & Sin problemas, pero me produce dolor \\
\hline & $\begin{array}{l}\text { Mi dolor tiene poco impacto en mis actividades sociales, excepto } \\
\text { aquellas más enérgicas (deportes) }\end{array}$ & & El dolor es intenso, pero logro viajes de hasta $2 \mathrm{~h}$ \\
\hline & Debido al dolor salgo muy poco & & Puedo viajar menos de $1 \mathrm{~h}$ por el dolor \\
\hline & Debido al dolor no salgo nunca & & Puedo viajar menos de 30 minutos por el dolor \\
\hline & No hago nada debido al dolor & & Solo viajo para ir al médico al hospital \\
\hline & >80 limitación funcional máxima & & Resultado de la evaluación \\
\hline & 60-80 discapacidad & & \\
\hline & 40-60 limitación intensa & & \\
\hline & 20-40 limitación moderada & & \\
\hline & 0-20 limitación funcional mínima & & \\
\hline
\end{tabular}


Tabla 7. Evidencia global de aplicación y análisis en la cirugía de columna.

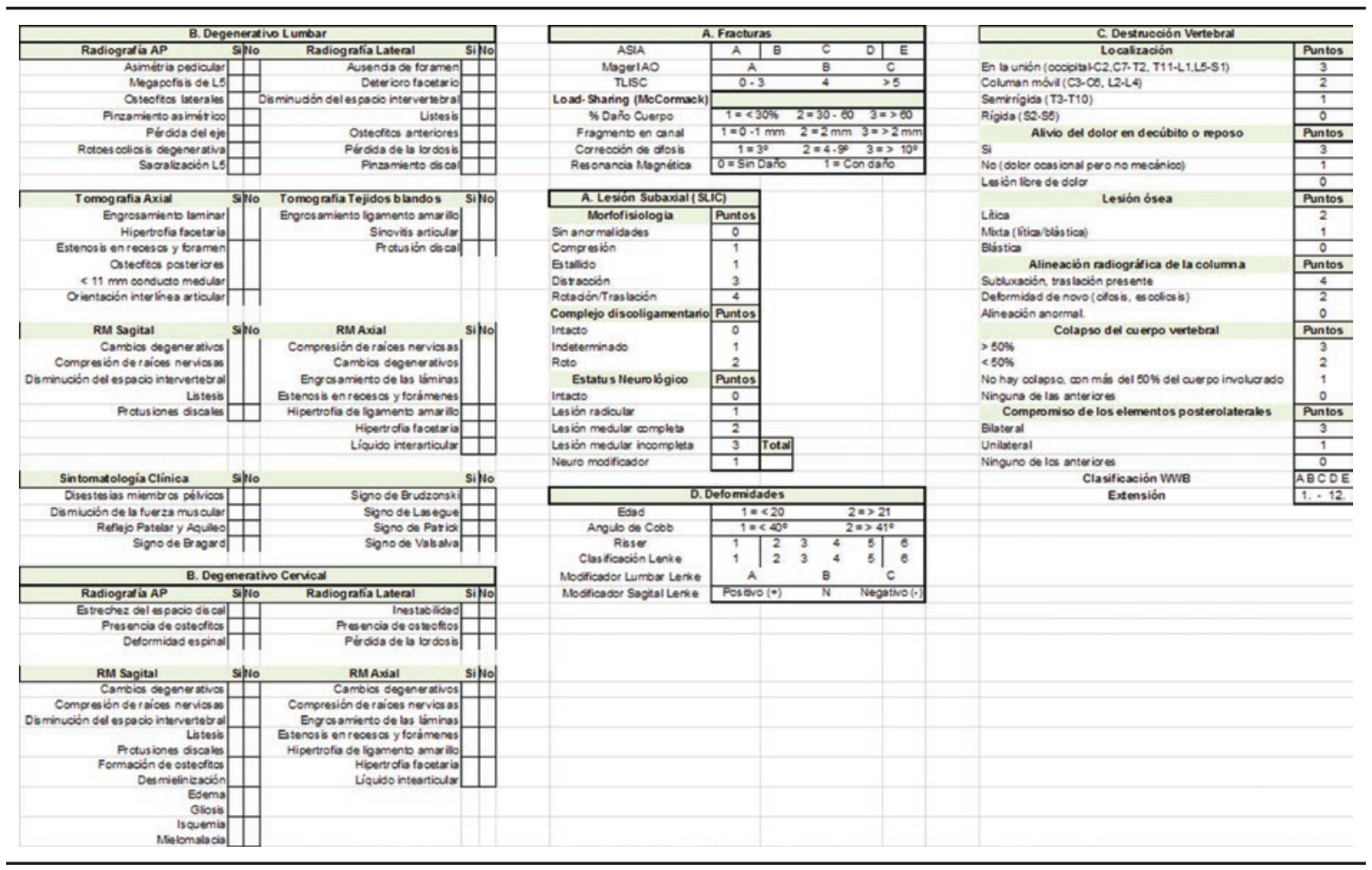

\section{Responsabilidades éticas}

Protección de personas y animales. Los autores declaran que para esta investigación no se han realizado experimentos en seres humanos ni en animales.

Confidencialidad de los datos. Los autores declaran que en este artículo no aparecen datos de pacientes.

Derecho a la privacidad y consentimiento informado. Los autores declaran que en este artículo no aparecen datos de pacientes.

\section{Financiamiento}

No se recibió ningún apoyo financiero para la realización de este estudio.

\section{Conflicto de intereses}

Los autores declaran no tener ningún conflicto de intereses con respecto a este artículo.

\section{Bibliografía}

1. Jiménez-Ávila JM, Rubio-Flores EN, González-Cisneros AC, Guzmán-Pantoja JE, Gutiérrez-Román EA. Directrices en la aplicación de la guía de práctica clínica en la lumbalgia. Cir Cir. 2018;86:29-37.

2. De la Cruz-Álvarez S, Canales-Nájera JA, Hurtado-Padilla A, Guevara-Villazón $\mathrm{F}$, Ledezma-Ledezma J. Corporectomía por acceso único posterior con colocación de caja expandible y fijación transpedicular en fracturas toracolumbares. Acta Ortopédica Mexicana. 2017;31:82-5.

3. Ames CP, Justin SS, Smith JS, Shay B, Scheer JK, Bess S, et al. Impact of spinopelvic alignment on decision making in deformity surgery in adults. J Neurosurg Spine. 2012;16:547-64.

4. Vaccaro AR, Schroeder GD, Kepler CK, Oner FC, Vialle LR, Kandziora F, et al. The surgical algorithm for the AOSpine thoracolumbar spine injury classification system. Eur Spine J. 2016;25:1087-94.

5. Feng Z, Liu Y, Yang G, Battié MC, Wang Y. Lumbar vertebral endplate defects on magnetic resonance images: classification, distribution patterns, and associations with modic changes and disc degeneration. Spine (Phila Pa 1976). 2018;43:919-27.

6. Urrutia J, Besa P, Campos M, Cikutovic P, Cabezón M, Molina M, et al. The Pfirrmann classification of lumbar intervertebral disc degeneration: an independent inter- and intra-observer agreement assessment. Eur Spine J. 2016;25:2728-33.

7. Modic MT, Steinberg PM, Ross JS, Masaryk TJ, Carter JR. Degenerative disk disease assessment of changes in vertebral body marrow with MR imaging. Radiology. 1988;166:193-9.

8. Fisher CG. A novel classification system for spinal instability in neoplastic disease. An evidence-based approach and expert consensus from the spine oncology study group. Spine (Phila Pa 1976). 2010;35:E1221-9.

9. Jiménez-Ávila JM, Cahueque-Lemus MA, Cobar-Bustamante AE, Bregni-Duraes MC. Vertebral destruction syndrome: from knowledge to practica. J Spine. 2015:4:1-4

10. Choi D, Crockard A, Tomita K. Review of metastatic spine tumour classification and indications for surgery: the consensus statement of the Global Spine Tumour Study Group. Eur Spine J. 2010;19:215-22.

11. Sponseller P, Flynn JM, Newton PO, Marks MC, Bastrom TP, Petcharaporn $\mathrm{M}$, et al. The association of patient characteristics and spinal curve parameters with Lenke classification types. Spine (Phila Pa 1976). 2012;37:1138-41

12. Falavigna A, Dozza DC, Teles AR, Wong CC, Barbagallo G, Brodke D, et al. Current status of worldwide use of patient-reported outcome measures (PROMs) in spine care. World Neurosurg. 2017;108:328-35.

13. Kim GM, Yi CH, Cynn HS. Factors influencing disability due to low back pain using the Oswestry Disability Questionnaire and the Quebec Back Pain Disability Scale. Physiother Res Int. 2015;20:16-21.

14. Fairbank JC, Pynsent PB. The Oswestry Disability Index. Spine (Phila Pa 1976). 2000;25:2940-52; discussion 2952.

15. Chiarotto A, Maxwell LJ, Terwee CB, Wells GA, Tugwell P, Ostelo RW. Roland-Morris Disability Questionnaire and Oswestry Disability Index: which has better measurement properties for measuring physical functioning in nonspecific low back pain? Systematic review and meta-analysis. Phys Ther. 2016;96:1620-37. 